\title{
ENSEÑAR APRENDER LA CIENCIA COMO INVESTIGACIÓN
}

\section{TEACH AND LEARN SCIENCE LIKE INVESTIGATION}

\author{
Raúl Ishiyama Cervantes ${ }^{1}$
}

\begin{abstract}
Aprender sin pensar es un esfuerzo perdido, pensar sin aprender, peligroso.

Yevqueni Ilin
\end{abstract}

\section{RESUMEN}

En el país se creía que la investigación científica la podian hacer solamente determinadas personas, ahora está al alcance de todos. En los últimos años se han creado terminologías y definiciones que "aseguran" la validez del proyecto y luego de los resultados; esto hizo que se crearan cursos sobre investigación, cada uno con su propia metodología. El Concytec estableció la Feria Escolar de Ciencia y Tecnología con el fin de despertar vocaciones por la investigación desde la escuela. Este trabajo se realizó con base a las ferias escolares entrevistando a alumnos y profesores de colegios; además a profesores de cursos de investigación en las universidades y profesionales que se graduaron con tesis. Se encontró que los alumnos de colegios tienen interés por la investigación, los estudiantes universitarios lo ven más complicado y consideran que investigar es difícil por los cursos detallistas sobre metodología de la investigación que les imponen, los que se gradúan con tesis demoraron en promedio un año en culminarla. Enseñar a realizar la investigación en forma natural y practica es amena para los estudiantes.

Palabras clave: Investigación, metodología, escolar, universitario.

\section{ABSTRACT}

In the country it was thought that the scientific research it could make only certain people, presently is within reach of all. In the last years terminologies and definitions have been created that "assure" the validity the project and after the results; it caused that courses on investigation were created, each one with its own methodology. The Concytec established the Scholastic Fair of Science and Technology with the purpose of waking up vocations by the investigation from the school. This work was made on base to the scholastic fairs interviewing to students and professors of schools; in addition to professors to courses of investigation in the universities and professionals who graduated with thesis. One was that the students of schools have interest by the investigation, the university students see it more complicated and consider to investigate is difficult by the detailed courses on methodology of the investigation that impose to them, those that graduate with thesis delayed in average a year in culminating it. Teaching to make the investigation in natural form and practice is pleasant for the students.

Key words: Investigation, methodology, student, and college student.

\section{INTRODUCCION}

La falta de vocación por la ciencia tiene su origen en parte en la escuela por la rutina de cumplir con enseñar lo que está en los libros. Se agrava en las zonas rurales en donde los alumnos cosas "raras" para ellos porque así lo exige el programa oficial único, cuando deberían aprender como utilizar la flora y la fauna así como conocer en detalle el medio ambiente de su región, para utilizar y proteger con el fin que redunde en su beneficio a largo plazo

Si se enseñara la ciencia como investigación en todos los niveles, seria positivo para el país, la sociedad y la persona. Las ferias escolares son esfuerzos del maestro actuando como asesor ante el entusiasmo científico de sus alumnos, no es parte del programa oficial.

Los programas universitarios a veces tienden a los extremos, por un lado una enseñanza tradicional del mire, dibuje y aprenda de memoria; por otro, la alta especialización a la que muy pocos pueden acceder por falta de laboratorios adecuados. Por otro lado en las universidades hay cursos "novedosos" sobre investigación científica cada uno con su propia metodología. Cursos eminentemente teóricos con muchas definiciones y clasificaciones que obligan a memorizar una serie de normas y conceptos. No se considera la investigación como la practica natural del ser humano, no se explota la curiosidad natural. La investigación científica requiere solamente de determinadas pautas; no se enseña, se aprende practicando bajo ciertas reglas para trabajar disciplinadamente.

Es alarmante la situación de la educación actual nadie hace nada en forma drástica para mejorar, desde la reforma educativa del gobierno militar que fue dejado de lado, se han realizado ensayos en cada gobierno, no hay un programa coherente para hacer investigación, el diario El Comercio informa que según el Concytec, sólo $0,1 \%$ del PBI se invierte en instigación y desarrollo cinético y tecnológico. El promedio latinoamerica-

${ }^{1}$ Universidad Peruana Cayetano Heredia 
no es $0,4 \%$ y varios países se han propuesto elevar su cuota hasta por lo menos $1 \%$. La UNI gasta en investigaciones 3,3 millones de soles por año. La Unión Europea ha dispuesto que la investigación científica aumente y ha puesto como regla para el 2010 el $3 \%$ del $\mathrm{PBI}$ se dedique a ella (1).

La investigación científica implica previamente el método científico, de proposiciones teóricas y de formas prácticas que sirven de directrices en su ejecución; de orientación filosófica y lógica; constituye una economía en el trabajo y una política que regula y equilibra las diversas etapas de la investigación.

La universidad es el lugar en donde primordialmente se deben formar los investigadores bajo pautas comunes. En el país existen 58 universidades reconocidas oficialmente 28 públicas y 30 privadas; además, cinco públicas y 16 privadas no institucionalizadas (1). Por su autonomía cada facultad tiene su propio reglamento para la elaboración de la tesis que son trabajos de investigación y cada asesor su propio concepto "original" sobre la investigación; en consecuencia hay gran diversidad para desarrollar ese trabajo que debería tener patrón con pautas comunes.

Los proyectos de investigación de los alumnos demoran semanas y a veces meses para ser aprobados, porque existen numeroso requisitos que deben cumplir, dicen que son para validar el proceso de la investigación, los investigadores calificados casi no lo aplican. (2).

Se gasta millones de soles en proyectos de investigación los que aparentemente quedan en proyectos porque no redunda en beneficio del país, se desconoce donde están los rsultados. Las revistas científicas en general no cumplen los requisitos para ser indizadas; de acuerdo al Scientific Citation Index (SCl) en el Perú en el año 2002 tuvo una producción de publicaciones de 0,90 por cada 100 habitantes, ocupando el primer lugar Chile con 15,00. En publicaciones peruanas mismo año destaca en primer lugar la Universidad Peruana Cayetano Heredia con 73 publicaciones, seguida por la Universidad Nacional Mayor de San Marcos con 20, la Pontifica Universidad Católica del Perú con 9, la Universidad Nacional de Ingeniería y el Instituto Geofísico con
7 cada uno. "Lo que se hace, si no se publica ni se difunde, es como si no existiera" (4).

\section{MATERIAL Y MÉTODOS}

La investigación se realizó con entrevista a profesores y alumnos de colegios que participaron en las ferias escolares de ciencia y tecnología en los últimos años; con ex alumnos que optaron hacer tesis para obtener el grado o título, con investigadores y profesores de metodología de la investigación.

\section{RESULTADOS}

Se observó que la enseñanza en los colegios es eminentemente memorística a pesar que los niños viven en una sociedad de informática, desean saber más de lo tradicional y buscan la libertad de trabajar adaptando el programa a sus necesidades cuando encuentran un profesor con la misma idea, aún con el peligro de ser castigado por tener iniciativa.

La Feria Escolar de Ciencia y Tecnología es promotora de la investigación científica en los últimos 15 años, ha logrado interesar a los escolares el interés por la investigación. Los maestros a pesar de no haber tenido formación para orientar en esta actividad hacen el esfuerzo para orientar a los estudiantes; lo grave es que de pronto le cambian las pautas sin explicación produciendo desconcierto. Es una actividad que prácticamente marcha sola, al inicio del año escolar lo alumnos por tradición buscan temas para trabajar y participar en la feria a nivel de su colegio, esto ocurre en casi todos los centros educativos del país.

Los estudiantes universitarios que han logrado participar en la investigación de sus profesores tiene la ventaja de aprender practicando. Los que no tuvieron esa practica y decidieron graduarse con tesis, demoraron en promedio un año. Pocos son los que siguen el esquema universal para presentar el informe final de la investigación, la mayoría tuvo que ceñirse a la exigencia de su Facultad.

No se sabe con precisión cuántos trabajos se han realizado en las ferias escolares, ni se ha hecho un seguimiento de los que llegaron a la final nacional, que podrían haber servido para benéfico del país con el apoyo de investigadores de la especialidad. Tampoco se sabe cuántas tesis se han realizado.

\section{DISCUSION}

El ser humano con su inteligencia trata de entender a la naturaleza con la finalidad de lograr mayor confort construyendo un mundo artificial. Los cambios que realiza lo logra gracias a la investigación científica. Mejor cuando el futuro investigador entiende que investigar es similar a "jugar". Todo juego es divertido se aprende a jugar practicando, cuanto más se practica se adquiere mayor habilidad. La pasión del joven es averiguar, investigar, descubrir, saber más.

Se aprende practicando en forma normal. La "profesión" del niño es jugar, en la escuela inicial se estimula el aprendizaje por el juego. A medida que crece se le inculcan ciertas reglas, Cuando llega a la escuela le imponen pautas pedagógicas y el aprendizaje por el juego se va diluyendo, como no entiende la razón de esa distorsión y del cambio en la metodología utiliza su memoria para demostrar que "asimila" la gran cantidad de información para poder responder en la evaluación o examen, luego lo olvida $(3,5)$

Es necesario desarrollar las armas intelectuales para el logro de la paz y la erradicación de la contaminación entre los muchos problemas. Esa arma es la educación, la verdadera educación, no las "verdaderas educaciones" con metodologías innovadoras complicadas e impuestas que no han servido a los estudiantes. Se debe utilizar la educación natural como la llamada "lengua materna".

Los conocimientos científicos van cambiando, al estudiante no se le dice por que cambian. Como cualquier persona el estudiante aprende solamente lo que él quiere aprender, lo demás lo memoriza para poder cumplir con responder las preguntas durante el examen a veces da la impresión que ser memorista es sinónimo de inteligente. Esto se debe a la falta de estímulo por la investigación por parte del profesor.

Una costumbre casi común en nuestro medio es que quien asume un cargo, lo primero que hace es "innovar" borrando casi todo lo que 
hizo su antecesor, elimina la colaboración de quienes conocen la actividad e "inventa" cosas personales sin consultar a masivamente a los maestros de aula quienes son los que viven la educación práctica, como tiene el poder siempre transitorio lo impone, el profesor se ve obligado a acatar. Satisface su ego con perjuicio de miles de estudiantes y profesores quienes se ven sometidos a cambios sin saber la razón (6).

Si se elimina los aspectos complicados con los que se rodea al proceso de la investigación científica de "alto nivel", nos quedamos con la investigación normal a la que cualquier persona disciplinada puede acceder. Si al pescado se le quita las escamas, las tripas y se le filetea, se tiene una excelente presentación de la carne del pescado. Si a los modelos sofisticados de enseñanza aprendizaje sobre investigación se le quita los adornos diseñado por los "expertos" que aparecieron en los últimos años, queda la enseñanza aprendizaje natural y simple.

La universidad ha sido creada para enseñar, y enseñar bien, con rol conciente dentro de la sociedad siendo su meta los fines de la educación para lograr el desarrollo intelectual del educando empleando la alta tecnología y recurriendo al ingenio cuando no se cuenta con recursos.

Hay formas de enseñar la ciencia, una es por la investigación. En los años sesenta causó impacto la Feria Científica Escolar, con esta actividad gran parte de los escolares de América Latina tuvieron y tienen la oportunidad de desarrollar sus inquietudes con relación a su curiosidad sobre temas científicos. En el Perú se inicia esta actividad en los años setenta por la Asociación Peruana para el Avance de la Ciencia (APAC) y en los ochenta el Concytec lo institucionaliza a nivel escolar, lo mismo que la Feria Universitaria con el fin de incentivar la investigación a ese nivel que no ha dado resultado por no tener continuidad. Al inicio en la feria escolar participaron unos cientos de estudiantes, luego miles, ahora son millones a nivel de los colegios. (3).

Los países en desarrollo requieren que los niños desde la escuela se autocuestionen y se pregunten el por qué de los hechos. Quienes tienen la tecnología educativa deben presentar una forma sencilla de enseñar, la tec- nología sofisticada no está al alcance de todos los maestros que trabajan en condiciones precarias.

Se debe luchar contra "Explícame en difícil, escribeme en obtuso"

En el sistema clásico de educación, el proceso del aprendizaje es la adquisición de conocimientos mediante determinados modelos de comportamiento. Los modelos adquiridos se obtienen de otros individuos de la misma especie, también pueden aprender individuos de otras especies (7).

El ser humano está programado genéticamente para aprender con mayor flexibilidad que cualquier otro animal, tiene la capacidad de abstracción y que le permite la conversión mental de los objetos y símbolos con contenidos significantes y convencionales que hacen posible la existencia del lenguaje.

El hombre no nace humano, nace con la capacidad de serlo por lo que tiene que aprender a ser humano. El aprendizaje consciente incluye las fases de ver, escuchar, observar y hacer. Cada cultura se diferencia por el énfasis que pone en una $u$ otra de estas fases.

Educación es el proceso sistemático de comunicación y aprendizaje mediante el cual se inculcan a los miembros de una sociedad determinada y

\section{REFERENCIAS BIBLIOGRAFICAS}

1. El Comercio. Prácticamente no hay fondos para la investigación científica. El Comercio 04-07-11, sección A, página 2.

2. Ishiyama Cervantes, Raúl. Editor. Feria escolar nacional de ciencia y tecnología Guía del participante. Lima: Concytec; 2001.

3. Ishiyama Cervantes, Raúl. Mecanismos de producción de la creatividad en ciencia y tecnología. En: Ciencia y tecnología para el desarrollo 2da edición. Lima: Concytec; 1989. pp. 179-183.

4. CONCYTEC. Perú ante la sociedad del conocimiento Indicadores de ciencia, tecnología e innovación 1960-2002. Lima: Concytec; 2003. pp.72-74.

5. Ishiyama Cervantes, Raúl. El semillero científico. En: Boletín Concytec 1998 julio; : 16-18.

6. Ishiyama Cervantes, Raúl . La enseñanza aprendizaje y la ética. En: Boletín Concytec 1997 julio: (6): 30-35.

7. Ishiyama Cervantes, Raúl; Hallasi Roselló, Dilma D. Audacia metodológica. En: 4to Encuentro Internacional Sobre Educación Productiva. Lambayeque: Universidad Nacional Pedro Ruiz Gallo facultad de Educación; 2002. pp.53-56.

8. El Comercio. Hablemos de educación. El Comercio 04-07-24, sección C, página 9.

E-mail: raishiyama@yahoo.com 